\title{
Decision aiding handbooks for managing contaminated food production systems, drinking water and inhabited areas in Europe
}

\author{
A.F. NISBET ${ }^{1}$, J. BROWN ${ }^{1}$, B.J. HOWARD ${ }^{2}$, N.A. BERESFORD ${ }^{2}$, \\ H. OLLAGNON ${ }^{3}$, C. TURCANU ${ }^{4}$, J. CAMPS ${ }^{4}, \mathrm{~K}_{\text {. ANDERSSON }}^{5}$, \\ A. RANTAVAARA ${ }^{6}$, T. IKÄHEIMONEN ${ }^{6}$, T. DURANOVA ${ }^{7}$, \\ D. OUGHTON $^{8}$, G. KIRCHNER ${ }^{9}$, C. PAPACHRISTODOULOU ${ }^{10}$, \\ K. IOANNIDES ${ }^{10}$, P. KWAKMAN ${ }^{11}$
}

\begin{abstract}
Three handbooks have been developed, in conjunction with a wide range of stakeholders to assist in the management of contaminated food production systems, inhabited areas and drinking water following a radiological incident. The handbooks are aimed at national and local authorities, central government departments and agencies, emergency services, radiation protection experts, the agriculture and food production sectors, industry and others who may be affected. The handbooks include management options for application in the different phases of an incident. Sources of contamination considered in the handbooks include nuclear accidents and radiological dispersion devices; the most relevant radionuclides are included. The handbooks are divided into several sections which provide supporting scientific and technical information; an analysis of the factors influencing recovery; compendia of comprehensive, state-of-the-art datasheets for around 100 management options and guidance on planning in advance. A decision-aiding framework comprising colour coded selection tables, look-up tables and decision trees and several worked examples are also included. The handbooks can be used as a preparatory tool, under non-crisis conditions, to engage stakeholders and to develop local and regional plans. The handbooks can also be applied as part of the decision-aiding process to develop a recovery strategy following an incident. In addition, the handbooks are useful for training purposes and during emergency exercises. To realise their full potential, the handbooks should be customised at national, regional and local levels.
\end{abstract}

\footnotetext{
Radiation Protection Division, HPA, Didcot, UK.

Centre for Ecology \& Hydrology, Lancaster, UK.

AgroParisTech, Paris, France.

SCK-CEN, Mol, Belgium.

RIS $\varnothing$ National Laboratory, Denmark.

STUK, Helsinki, Finland.

VUJE, Slovak Republic.

The Norwegian University of Life Sciences, Ås, Norway.

BfS, Salzgitter, Germany.

University of Ioannina, Greece.

11 RIVM, The Netherlands.
} 


\section{Introduction}

The accident at the Chernobyl Nuclear Power Plant prompted a number of initiatives to document and evaluate options for managing the effects of this type of radiological emergency. Information on these management options has been recorded in a systematic way using a datasheet format. Compendia of these datasheets have been published for the management of contaminated food production systems, drinking water supplies and inhabited areas that cover all phases of accident response (Andersson et al., 2003; Nisbet et al., 2004; Beresford et al., 2006). However, only subsets of these options are likely to be applicable depending on the timing and scale of application, types of radionuclides released and local management practices. Consequently, a need was identified to develop handbooks to aid decision making, not just from a scientific and technical point of view but also taking into account stakeholder opinion. In response, handbooks for food production systems and inhabited areas were developed during a series of UK and European initiatives, the latter supported by the EC through the EURANOS integrated project. In 2005, the first version of the UK Recovery Handbook for Radiation Incidents was published (HPA, 2004). Since then, there has been an ongoing and iterative process involving stakeholder groups and networks such as FARMING (Nisbet et al., 2005) to develop generic European handbooks for food production systems and inhabited areas, the first versions of which were published in 2006 and 2007 respectively (Nisbet et al., 2006; Brown et al., 2007). Eight emergency centres in Member States that had not been involved in the development of these handbooks took part in demonstration activities to establish whether the handbooks would be useful for the purposes of contingency planning and accident management (Nisbet et al., 2010). Use was made of emergency exercises or similar events based on scenarios involving contamination of the foodchain and inhabited areas. Feedback from all of the demonstrations was positive with constructive criticism given on how to improve the navigation, structure and format of the handbooks. All of the key improvements highlighted during the demonstrations were taken into account and included in a revised version of the handbooks (Nisbet et al., 2009, in prep.); drinking water became the topic of a standalone handbook at this time (Brown et al., 2009). An overview of the updated handbooks is presented in this paper.

\subsection{Objectives and scope}

The handbooks have been developed to:

- provide up-to-date information on management options for reducing the consequences of contamination of the foodchain, drinking water supplies and inhabited areas; 
- outline the many factors that influence the implementation of these options;

- provide guidance on planning in advance of an incident;

- illustrate how to select and combine management options and hence build a recovery strategy.

The sources of contamination considered in the handbooks are from a nuclear site or weapons' transport accident. However, many of the management options described will also be relevant to other radiation incidents (e.g. an improvised terrorist device), even though the pattern of contamination would be different. The phases covered by the handbooks are the pre-deposition to post-accident phases, with emphasis on recovery in the post-accident phase. The handbook for food production systems considers agricultural and domestic production as well as the gathering of wild foods. The handbook for inhabited areas focuses on the different surfaces present, i.e. buildings; road and paved areas; soils and grass; trees and shrubs. The handbook for drinking water, which was originally part of the inhabited areas handbook, considers public and private water supplies.

\subsection{Audience and application}

The handbooks are specifically targeted at national and local authorities; central government departments and agencies; emergency services; experts in radiation protection; representatives from agriculture and food production sectors; representatives from industry, including the water utilities; and other stakeholders who may be affected or concerned, depending on the situation.

The handbooks can be considered solely as reference documents containing well focused and generic state-of-the-art information on scientific, technical and societal aspects relevant to the management of contaminated areas. However, when used in isolation (i.e. not as part of a project involving a stakeholder participatory process), the full potential of the handbooks cannot be realised. In the same way that these handbooks were developed through a process of stakeholder participation, they are intended to be applied using a similar participatory approach. Examples of the most likely applications of the handbooks are in:

- the preparation phase, under non-crisis conditions, to engage stakeholders and to develop local, regional and national plans/framework/tools;

- the post-accident phases, for use by local and national stakeholders as part of the decision-aiding process;

- training;

- preparation for, and during, emergency exercises. 


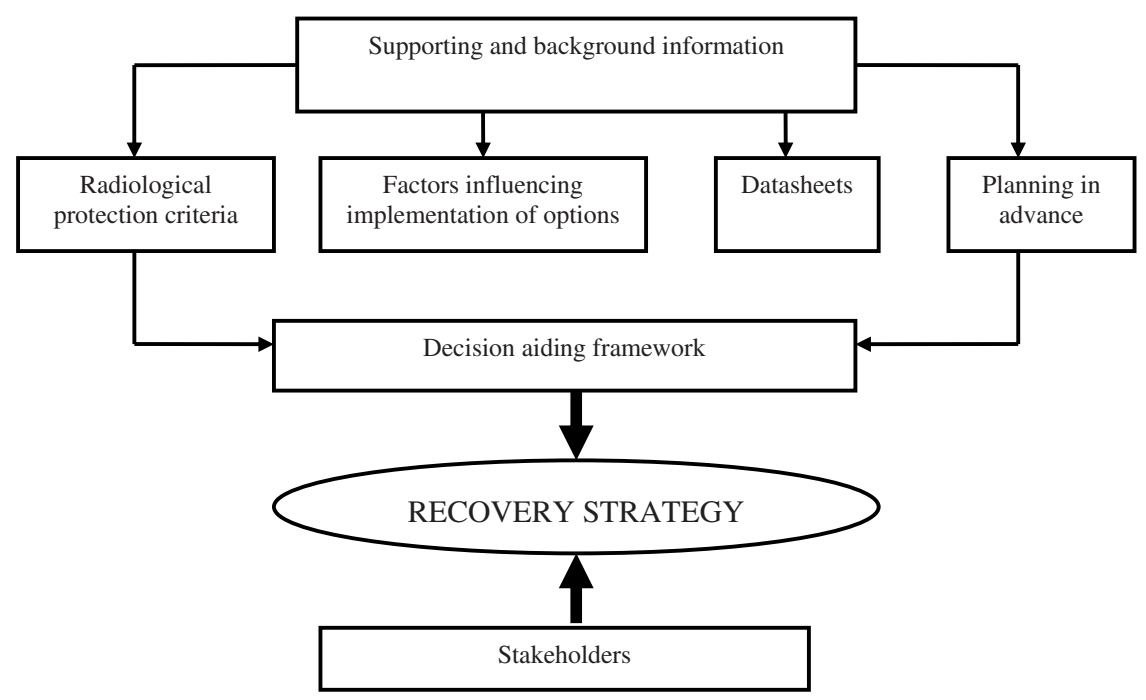

Figure 1 - Generalised structure of the handbooks.

\subsection{Structure and content}

The overall structure of the three handbooks is similar and presented in Figure 1. Supporting and background information is provided in appendices. The initial sections provide information on radiation protection criteria, factors influencing the implementation of management options and datasheets for individual management options. Guidance on what can be done on planning in advance of an incident is also given. There is a main decision aiding framework, comprising selection tables, decision trees and look-up tables to assist the user in developing a management strategy based on selecting and combining options on a site-specific basis. Each handbook includes several worked examples that illustrate how to apply the decision aiding framework for a range of accident scenarios. The handbooks should be used as part of a participatory process involving local and national stakeholders in the development of a recovery strategy (i.e. lower segment of Fig. 1).

\section{Management options}

\subsection{Purpose}

The primary aim of a management option is to reduce doses either from the consumption of contaminated foodstuffs and water via the ingestion pathway or 
from exposure to contaminated surfaces in an inhabited area via external irradiation and inhalation of resuspended material. A secondary objective is to provide reassurance to consumers and people living in contaminated areas.

\subsection{Datasheet template}

The handbooks consider more than 100 management options; 58 for food production systems, 57 for inhabited areas and 6 for drinking water. There is a large amount of information on each of these management options which needs to be considered before a decision can be made on the most appropriate option(s) to select. A datasheet template was designed to systematically record information in a standardised format, taking into account most of the criteria that decision makers might wish to consider when evaluating different options (Tab. I). The template includes a short description of the option, its key attributes, constraints, effectiveness, feasibility, the waste generated, the types of incremental doses incurred, costs, side effects, and a summary of practical experience of implementing the option. The format and content of the datasheets are based largely on similar documents developed initially in the STRATEGY project (Anderson et al., 2003; Howard et al., 2004; Nisbet et al., 2004), and which were updated and expanded for additional radionuclides, additional management options, and broader timescales for implementation (Beresford et al., 2006)).

\subsection{Classification of management options}

Management options can be implemented at different phases of the response extending from the pre-deposition stage and continuing for the days, weeks, months and even years after the accident. In food production systems, management options are designed to target particular media and contamination pathways including soil, crops, livestock and other animal products. They are not only aimed at addressing health concerns but also a wide range of other issues such as the local economy, societal and ethical concerns and disposal of wastes.

Management options for inhabited areas can be divided into two main groups: options that shield people from the contamination (shielding options) and those that remove contamination (removal options, also called decontamination or clean-up options). The implementation of management options is generally the responsibility of the authorities; however self-help options, which may be implemented by the affected population, can also be useful. Importantly, the option not to carry out any recovery can also be a valid alternative. Management options may be further classified according to the surface that they are targeted at (i.e. buildings; road and paved areas; soils and grass; trees and shrubs). 


\section{TABLE I}

Information presented in the datasheets of management options.

\begin{tabular}{|c|c|}
\hline $\begin{array}{l}\text { Key information about the management } \\
\text { option }\end{array}$ & $\begin{array}{l}\text { Objective. } \\
\text { Other benefits. } \\
\text { Management option description. } \\
\text { Target. } \\
\text { Targeted radionuclides. } \\
\text { Scale of application. } \\
\text { Contamination pathway. } \\
\text { Exposure pathway pre-intervention. } \\
\text { Time of application. }\end{array}$ \\
\hline Constraints & $\begin{array}{l}\text { Legal. } \\
\text { Social. } \\
\text { Environmental. }\end{array}$ \\
\hline Effectiveness & $\begin{array}{l}\text { Effectiveness of management option in reducing contamination; } \\
\text { Factors influencing effectiveness of procedure. }\end{array}$ \\
\hline Feasibility & $\begin{array}{l}\text { Required specific equipment. } \\
\text { Required ancillary equipment. } \\
\text { Required utilities and infrastructure. } \\
\text { Required consumables. } \\
\text { Required skills. } \\
\text { Required safety precautions. } \\
\text { Other limitations. }\end{array}$ \\
\hline Waste & $\begin{array}{l}\text { Amount and type. } \\
\text { Possible transport, treatment and storage routes. } \\
\text { Factors influencing waste issues. }\end{array}$ \\
\hline Doses & $\begin{array}{l}\text { Incremental doses. } \\
\text { Reduction in doses. }\end{array}$ \\
\hline Intervention costs & $\begin{array}{l}\text { Equipment. } \\
\text { Consumables. } \\
\text { Operator time. } \\
\text { Factors influencing costs. } \\
\text { Compensation costs. } \\
\text { Waste costs. } \\
\text { Assumptions. } \\
\text { Communication needs. }\end{array}$ \\
\hline Side effect evaluation & $\begin{array}{l}\text { Ethical considerations. } \\
\text { Environmental impact. } \\
\text { Agricultural impact. } \\
\text { Social impact. } \\
\text { Other side effects. } \\
\text { Stakeholder opinion. }\end{array}$ \\
\hline \multicolumn{2}{|l|}{ Practical experience } \\
\hline \multicolumn{2}{|l|}{ Key references } \\
\hline Comments & \\
\hline
\end{tabular}

Management options for drinking water are targeted at public and private water supplies and can be divided into two main groups: options that provide clean water through alternative supplies and changes to the source of the supply and those that involve the treatment of water in some way. 


\section{Factors influencing implementation of management options}

The characteristics of the radionuclides deposited into the environment from an incident, as well as the type of land that is affected by the contamination and what it is used for, have a significant influence on the selection of management options. The implementation of these management options is not trivial. There are a number of factors that need to be taken into account when developing a management strategy for the long term recovery of contaminated areas. The most important of them are:

- temporal and spatial factors;

- effectiveness of management options;

- waste disposal issues;

- radiological impact (protection of workers);

- environmental impact;

- economic cost;

- societal and ethical aspects;

- communication and information issues.

Each factor is considered in detail in the handbooks both as standalone sections and incorporated into individual datasheets. Some of the key issues are highlighted below.

\subsection{Temporal and spatial factors}

The selection of management options should take account of temporal factors such as the time of year when the incident happened, the variation in activity concentrations of the deposited radionuclides with time and their movement through the environment. When selecting management options, it is also helpful to consider them according to the timescale of their implementation. In the short term, prompt actions are necessary $e . g$., when dairy cattle are grazing outdoors at the time of deposition, leafy green vegetables are ready for harvest and when mushrooms and berries are available for gathering. However, many actions take time to organise and prepare (e.g. provision of uncontaminated animal feed, distribution of feed additives, topsoil and turf removal). For less urgent situations (e.g. livestock not ready for slaughter, immature crops in the field), several weeks are available in which to decide on and implement appropriate management options. Some situations may require rather drastic or irreversible actions such as change of land use or demolishing buildings. In such situations, stakeholder dialogue and consultation will be essential and sufficient time must be allowed (i.e. months) for the process to be fully implemented. 
Management options also need to be selected on the basis of the size of the area affected, levels of contamination present and land use. Typically, there will be areas where contamination levels are very high and priority has to be given to the direct protection of the population (e.g. by sheltering and evacuation). In these areas, protective measures for agricultural production should be considered as a low priority. In other areas not subject to emergency countermeasures, restrictions on the entry of food into the foodchain may be required. Levels of contamination in food products in these areas can be reduced by implementing a suitable set of management options. Finally, there will be other areas not contaminated (e.g. regions adjacent to contaminated areas) but which could still be affected indirectly. In this case, there would be a requirement for extra monitoring to maintain public confidence.

\subsection{Effectiveness}

The management options considered in the handbooks are designed to reduce the (i) ingestion dose from the consumption of contaminated foodstuffs and drinking water; (ii) external dose rates from deposited radionuclides; and (iii) inhalation dose from resuspended material. Consequently, the handbooks have to be used in conjunction with measurement data and models to estimate, for example, the deposition of radioactive material on relevant surfaces and transfer in the food chain.

Information on effectiveness is generally presented in the handbooks as a percentage reduction in activity concentration in the target medium (i.e. soils, crops, building surfaces, roads) after implementing the option. There are some management options, however, which may be considered more as supporting measures (e.g. provision of monitoring equipment and live monitoring), which can increase the effectiveness of other options as well as providing reassurance, but which may not directly reduce doses. Effectiveness is influenced by both technical and societal criteria including application rates, duration of treatments, physical and chemical form of the radionuclide, biological half-live, timeliness of implementation, compliance and acceptability.

\subsection{Waste disposal issues}

The implementation of management options can generate wastes that need to be managed. For example, considerable volumes of contaminated waste can be generated as a result of the placing of restrictions on the marketing of crops, milk and meat. As these restrictions are based on statutory requirements it is essential that appropriate routes of disposal be identified in advance of future accidents or incidents. However, it is not just the placing of restrictions on foodstuffs that creates wastes; several management options also produce contaminated by-products 
(e.g. slaughtering dairy cows, topsoil removal, firehosing buildings, water treatment facilities) and routes for their disposal must be considered when the option is selected. The handbooks provide datasheets and descriptions of disposal options for waste arising from food production systems and inhabited areas, respectively.

In inhabited areas, contaminated waste will also arise whether or not management options are implemented. These wastes arise from:

- contaminated refuse and goods;

- waste water from rainfall and run-off.

In assessing the impact of waste on public, workers and the environment, it is important to consider contamination levels in the waste, quantities of waste produced and doses to workers and members of the public from exposure to the waste.

\subsection{Radiological impact}

Radiological impact refers to the incremental doses received as a result of implementing management options, which are not part of normal practices. Incremental doses are most likely to be received by implementers (e.g. farmers and those involved in clean-up). A number of factors influence the magnitude of the doses received including: type of radionuclide released, medium that has been contaminated, exposure pathways resulting from carrying out the management option (external, inhalation, ingestion, irradiation of the skin), length of exposure time, generation of secondary wastes and additional doses from disposal.

\subsection{Environmental impact}

Environmental impacts may occur when management options are carried out in food production systems and inhabited areas. The impacts can be positive or negative, direct or indirect. Direct environmental impacts can include changes in biodiversity, soil fertility and structure, and enhanced soil erosion as well as pollution of air and water. Indirect effects happen, for example, when an individual's freedom is reduced by changes to traditional lifestyles, or where the utility of the environment changes through restricting access. Information on the environmental impact of each management options is given in the datasheets in each handbook.

\subsection{Economic cost}

It is difficult to predict the economic cost of implementing management options because of the numerous factors that influence cost (Alvares-Farizo et al., 2009). 
There are direct costs such as those incurred through loss of production, closure of businesses, provision of temporary premises; cost of implementation: labour, consumables, equipment, transport; cost of handling of wastes and carrying out environmental remediation. Indirect costs are equally important, although they are inherently more difficult to quantify. Examples are loss of market share, regional impact on tourism which could extend beyond the affected area; and impact on society when people cannot return to normal lifestyles.

\subsection{Societal and ethical issues}

Societal and ethical factors are relevant to the management of contaminated areas. When deciding which management option should be carried out it is important to understand the implication of any actions on the population, to take into account individual and community concerns and to recognise the need to involve local stakeholders in the identification of problems and their solution. Some management options potentially have a negative impact on society by causing disruption (e.g. through restricting access or activities), anxiety and stress (e.g. by causing panic, upheaval), and stigma (e.g. by affecting businesses or tourism). These need to be balanced against positive impacts through provision of reassurance and improvements to living conditions. There are also ethical issues to be addressed when selecting management options, including: provision of selfhelp options that reinforce liberty and dignity; the distribution of doses over space and time and between different members of the community, animal welfare, environmental risk and consequences for future generations.

\subsection{Communication and information issues}

The provision of information, and how that information is communicated, will have a significant influence on how the authorities manage the situation, on society's response to the problem and on the overall success of the recovery strategy. Maintaining public confidence is paramount. Trust is fragile, easy to lose and notoriously difficult to develop and/or regain once lost. Past experience has shown that it is important to develop a framework for information and communication strategies under non-crisis conditions. It should involve stakeholders due to the complexity of issues. The type of information disseminated should be tailored to meet a variety of needs. The form of communication should be adapted to different levels of understanding, circumstances and to address issues at stake. This should all happen in parallel with the development of the management strategies and at all stages of the response. The handbooks do not specifically include a detailed communication strategy or pre-prepared press statements. 
TABLE II

Eight step process for selecting and combining management options.

\begin{tabular}{ll}
\hline Step & Action \\
\hline 1 & $\begin{array}{l}\text { Identify one or more food production systems or inhabited area surfaces that are likely to be/have } \\
\text { been contaminated }\end{array}$ \\
\hline 2 & $\begin{array}{l}\text { Refer to selection tables for specific production systems or inhabited surfaces. These selection } \\
\text { tables provide a list of all of the applicable management options for the production system/surface } \\
\text { selected }\end{array}$ \\
\hline 3 & $\begin{array}{l}\text { Refer to look-up tables showing applicability of management options for each radionuclide being } \\
\text { considered }\end{array}$ \\
\hline 4 & $\begin{array}{l}\text { Refer to look-up tables showing checklists of key constraints for each management option } \\
5\end{array}$ \\
\hline 6 & $\begin{array}{l}\text { Refer to look-up tables showing effectiveness of each option } \\
\text { Refer to individual datasheets for all options remaining in the selection table and note the relevant }\end{array}$ \\
\hline 7 & $\begin{array}{l}\text { Based on the outputs from Steps 1-7, select and combine options that should be considered as part } \\
\text { of the recovery strategy }\end{array}$ \\
\hline
\end{tabular}

\section{Decision-aiding framework}

In the event of a radiological accident or incident, decision-makers will need to be in a position to construct a strategy for managing contaminated food production systems, inhabited areas and drinking water supplies. For small-scale, single radionuclide releases, the strategy may comprise of one or two management options that could be applied over the first few days or weeks following the accident. For wide scale, multi-radionuclide releases, a management strategy is likely to be more complex, comprising a series of management options that could be implemented over different phases of accident response.

The handbooks provide information on more than 100 management options that could assist in the recovery of food production systems, inhabited areas and drinking water supplies. The selection of individual options depends on a wide range of criteria and only a subset of options will be applicable for any one accident scenario. As each accident is likely to be different in terms of its radiological composition, it is not possible to devise a generic strategy. Consequently an eight-step process for selecting and combining options was developed for inclusion in the handbooks (Tab. II). Colour coded selection tables provide a focus for developing a recovery strategy and a series of look up tables are presented to help eliminate options. Each selection table lists all of the applicable management options for each type of food production system or surface within an inhabited area. They provide an indication of whether the management options are suitable for implementation in the pre-deposition, early, medium or late 
phases. They also provide an indication of whether the management options are likely to be implemented based on knowledge of potential technical, logistical, economic or social constraints. The colour-coding distinguishes between options that would:

- usually be justified or recommended having few if any constraints;

- also be recommended but would require further analysis to overcome potential constraints;

- have to undergo a full analysis and consultation with stakeholders before implementation because of serious economic or social constraints, and options that would only be justified in specific circumstances following full analysis and consultation due to major technical or logistical constraints.

The classification used in the selection tables is intended to be a guide and requires customisation at local or regional level by the relevant stakeholders. The colourcoded selection tables are also available in grey scale for users with colour-vision deficiency and for printing purposes. An example of a selection table to assist in the selection and combining of options for contaminated roads and paved areas is given in Table III. Look-up tables assist in the elimination of options according to the specifics of the incident, for example, according to the radionuclides released, site specific constraints and volumes of waste generated. A single decision tree is used instead of a selection table in the handbook for drinking water as there are fewer options to consider.

\section{Planning in advance}

The response to the effects of a major accident or emergency is managed primarily at the local level using emergency plans drawn up in advance. These are easily applied and are universally accepted. Emergency plans do not include actions to be taken in the post-emergency phase (i.e. recovery phase) for which it is much more difficult to be prescriptive about actions to take due to variations in local circumstances. Nevertheless, there should be planning for recovery up to the design basis accident, albeit in much less detail. Although much depends on the nature of the emergency or incident (e.g. its magnitude and the extent of radioactive contamination), consideration of topics such as "requirements for information" and "outline arrangements" prior to the occurrence of an incident should improve the speed of recovery response and may also ensure a more successful outcome. The handbooks provide a breakdown of topics covering data and information requirements that could usefully be gathered in advance of an incident. Consideration needs to be given to the development and sharing of localised databases on commercial and private food producers, dietary habits, businesses, suppliers of raw materials, contractors, waste disposal facilities, and 
TABLE III

Illustrative selection table of management options for roads and paved areas.

\begin{tabular}{lcc}
\hline When to apply & $\begin{array}{c}\text { Early } \\
\text { (days - weeks) }\end{array}$ & $\begin{array}{c}\text { Medium-long term } \\
\text { (months - years) }\end{array}$ \\
\hline
\end{tabular}

Restrict access

Permanent relocation from residential areas

Prohibit public access to non-residential areas

Restrict workforce access (time or personnel) to nonresidential areas

Temporary relocation from residential areas

Removal and shielding options

Firehosing

High pressure hosing

Snow removal

Surface removal and replacement

Tie down (fixing contamination to the surface) bitumen (permanent)

Tie down (fixing contamination to the surface) - sand (temporary)

Tie down (fixing contamination to the surface) - water (temporary)

Turning paving slabs

Vacuum sweeping

Key:

\begin{tabular}{|l}
\hline Recommended with few constraints \\
\hline Recommended but requires further evaluation to overcome some constraints \\
\hline Economic or social constraints exist, requiring full analysis and consultation period \\
\hline $\begin{array}{l}\text { Technical or logistical constraints may exist, or the option may only be appropriate on a site } \\
\text { specific basis }\end{array}$ \\
\hline
\end{tabular}

other information. Some of these databases may already exist, but even then, it is not always widely known who would be the point of contact and who would have responsibility for maintaining the databases (the type of information stored could rapidly become out-of-date). The handbooks also give a list of factors that might need to be considered when developing outline arrangements for a recovery strategy, focussed at the local level, in advance of an incident. This includes development of a generic strategy, recovery criteria, roles and responsibilities, stakeholder involvement, protection of workers, practicability of management options. 
There is a broad diversity of climatic conditions, food production systems, buildings, culture, infrastructure and regulatory frameworks across Europe. Planning for recovery in advance of an incident will require customisation of the generic handbooks at national, regional or local level. Customisation also provides a good opportunity to review national documents and guidelines and to update and to incorporate this information into national versions of the handbooks. An essential component of the customisation process is the involvement of all stakeholders to better identify and include national/regional/local specificities. National, regional and local stakeholder processes should continue, and where none exist they should be initiated at the planning stage, under non-crisis conditions as part of familiarisation.

\section{Conclusions}

Three handbooks have been developed to assist in the selection of management options for contaminated food production systems, inhabited areas and drinking water in Europe. A wide range of stakeholders provided scientific, technical and practical input. A decision-aiding framework comprising colour coded selection tables, look-up tables and decision trees are included to assist with the elimination of options, together with several worked examples. The handbooks can be used as a preparatory tool, under non-crisis conditions to engage stakeholders. They can also be applied as part of the decision-aiding process to develop a recovery strategy following an incident. In addition, the handbooks are useful for training purposes and during emergency exercises. To realise their full potential the handbooks should be customised at national, regional and local levels in conjunction with stakeholders.

Acknowledgements. Work on the handbooks received partial financial support from the European Commission Sixth Framework Programme (Nuclear Fission/Radiation Protection) under the EURANOS integrated project: European approach to nuclear and radiological emergency management and rehabilitation strategies (Contract No: FI6R-CT-2004-508843).

\section{REFERENCES}

Alvarez-Farizo B., Gil J.M., Howard B.J. (2009) Impacts from restoration strategies: assessment through valuation workshops, Ecol. Econ. 68, 287-297.

Andersson K.G., Roed J., Eged K., Kis Z., Voigt G., Meckbach R., Oughton D.H., Hunt J., Lee R., Beresford N.A., Sandalls F.J. (2003) Physical countermeasures to sustain acceptable living and working conditions in radioactively contaminated residential areas. Riso-R-1396 (EN), Riso national laboratory, Denmark.

Beresford N.A., Barnett C.L., Howard B.J., Rantavaara A., Rissanen K., Reales N., Gallay F., Papachristodoulou C., Ioannides K., Nisbet A.F., Brown J., Hesketh N., Hammond D., Oatway W., 
Oughton D., Bay I., Smith J.T. (2006) Compendium of countermeasures for the management of food production systems, drinking waters and forests. Available at http://www.euranos.fzk.de.

Brown J., Hammond D.J., Kwakman P. (2009) Generic handbook for assisting in the management of contaminated drinking water in Europe following a radiological emergency. EURANOS(CAT1)-TN(09)-02.

Brown J., Mortimer K., Andersson K., Duranova T., Mrskova A., Hänninen R., Ikäheimonen T., Kirchner G., Bertsch V., Gallay F., Reales N. (2007) Generic handbook for assisting in the management of contaminated inhabited areas in europe following a radiological emergency. EURANOS(CAT1)-TN(07)-02. Available at http://www.euranos.fzk.de.

Howard B.J., Liland A., Beresford N.A., Andersson K.G., Cox G., Gil J.M., Hunt J., Nisbet A., Oughton D., Voigt G. (2004) A Critical Evaluation of the STRATEGY Project, Radiat. Prot. Dosim. 109, 63-67.

HPA (2005) UK Recovery Handbook for Radiation Incidents. HPA-RPD-002 (ISBN 0-85951-559-1). Available at http://www.hpa.org.uk/radiation.

Nisbet A.F., Andersson K.G., Duranova T. (2010) Demonstration of generic handbooks for assisting in the management of contaminated food production systems and inhabited areas in Europe, Radioprotection 45, S243-S249.

Nisbet A.F., Brown J., Rochford H., Cabianca T., Jones A., Andersson K., Duranova T., Hänninen R., Ikäheimonen T., Kirchner G., Bertsch V., Gallay F., Reales N. (in prep.). Generic handbook for assisting in the management of contaminated inhabited areas in Europe following a radiological emergency $v 2$.

Nisbet A.F., Jones A., Turcanu C., Camps J., Andersson K.G., Hänninen R., Rantavaara A., Solatie D., Kostiainen E., Jullien T., Pupin V., Ollagnon H., Papachristodoulou C., Ioannides K., Oughton D. (2009) Generic Handbook for Assisting in the Management of Contaminated Food Production Systems in Europe following a radiological emergency v2. EURANOS(CAT1)TN(09)-01. Available at http://www.euranos.fzk.de.

Nisbet A.F., Mercer J.A., Hesketh N., Liland A., Thørring H., Bergan T., Beresford N.A., Howard B.J., Hunt J., Oughton D.H. (2004) Datasheets on countermeasures and waste disposal options for the management of food production systems contaminated following a nuclear accident. Chilton, NRPB-W58.

Nisbet A.F., Mercer J.A., Rantavaara A., Hanninen R., Vandecasteele C., Carlé B., Hardeman F., Ioannides K.G., Papachristodoulou C., Tzialla C., Ollagnon H., Jullien T., Pupin V. (2005) Achievements, difficulties and future challenges for the FARMING network, J. Environ. Radioact. 83, 263-274.

Nisbet A.F., Rice H., Jones A., Jullien T., Pupin V., Ollagnon H., Hardeman F., Carlé B., Turcanu C., Papachristodoulou C., Ioannides K., Hänninen R., Rantavaara A., Solatie D., Kostiainen E., Oughton D. (2006) Generic handbook for assisting in the management of contaminated food productions systems in Europe following a radiological emergency, EURANOS(CAT1)TN(06)-06. Available at http://www.euranos.fzk.de. 